Int. J. Dev. Biol. 48: 271-274 (2004)

Essay

\title{
Constant and continuous growth reduction as a possible cause of ageing
}

\author{
ETIENNE GERAERT* \\ Vakgroep Biologie, Universiteit Gent, Belgium
}

\begin{abstract}
Post-embryonic growth is characterized by a constant reduction of some growth parameters in relation to other growth parameters. Comparison of growth in chickens, rats and nematodes reveals an identical growth pattern, so a theory about the growth process in general is presented. It is presumed that the same growth promoting and growth inhibiting substances regulate not only growth but also ageing and that it is the equilibrium between growth promoters and growth inhibitors which is constantly changed.
\end{abstract}

KEY WORDS: growth promoter, growth inhibitor, rat skull, chicken, allometry, parabolic curve

\section{Introduction}

Slack (1999) mentioned 'three very interesting questions associated with growth: (1) what controls the absolute size of the whole, or why are we bigger than mice? (2) Within a whole, what maintains the constancy of proportions of individual parts? (3) How is a possible change of relative proportions (allometry) produced? 'Bartoletti's et al. (1999) first sentence on the contrary reads ' The model of allometric growth (Jolicoeur, 1963; Hills, 1982; Klingenberg, 1996) assumes that various parts of an organism grow at a constant relative rate'. A different approach to growth is apparently needed. This article presents one such possibility.

Growth occurs as well in unicellular as in multicellular organisms. It is only in multicellular organisms that growth can be considerable; these organisms die, so multicellularity is only temporarily an advantage. The general idea is that growth starts exponentially (Huxley, 1924) till adulthood is reached, then unicellular offspring is made and afterwards ageing starts. Some presume that ageing is caused by the activity of special genes that were not active during growth. If these ageing genes were to be stopped, the adult would remain young and vigorous. Exponential growth means growth without restrictions; if, however, we consider that multicellularity in itself causes problems we might as well assume that the restrictions start at the very beginning. When a zygote divides, the several cells become slightly different; every cell has its own metabolism: some products of each cell positively or negatively influence the metabolism of each other cell (see e.g. the Chapter Cell Communication in Alberts et al., 2002). As a consequence a multicellular organism produces various growth promoting and growth inhibiting substances influencing the growth patterns.
To simplify the study of the three-dimensional and complicated growth patterns it is customary to only measure some distances (or other data) and to compare the measurements. By putting observed values of a growth pattern in an arithmetic graph, curved lines are often found. The nature of such a curve has been a subject of controversy for years; Huxley (1924) proposed an exponential curve; as this curve was not able to explain why growth stops several other curves and variants have been introduced (review in Zeger and Harlow, 1987 for the curves known then). Some of these curves describe growth patterns different from what is studied here: e.g. West et al. $(1997,2001)$ argue for fractional power laws on the basis that the limiting factor in growth is the formation of branching trees of the circulatory system and that this has an essentially fractal dimensionality.

The study of post-embryonic growth in various nematodes (Geraert, 1978a, 1978b, 1979) showed that several morphological parameters are closely related to each other, a relationship that was best expressed in a quadratic formula. To establish whether that approach is also applicable to growth in other animals, data have been gathered from the literature; studies in which growth was investigated in one and the same population from young to adult stage were favoured.

\section{Results}

\section{Rat skull}

In Fig. 1 the total skull length is compared to four distances on the skull, which show a progressing reduction in growth. The most interesting of them is the cribriform plate of which the width diminishes long before adulthood is reached. For each comparison a quadratic parabola has been calculated and added to the

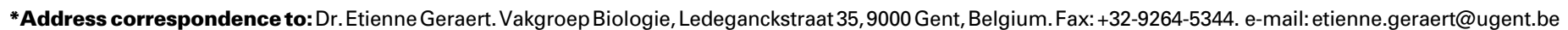




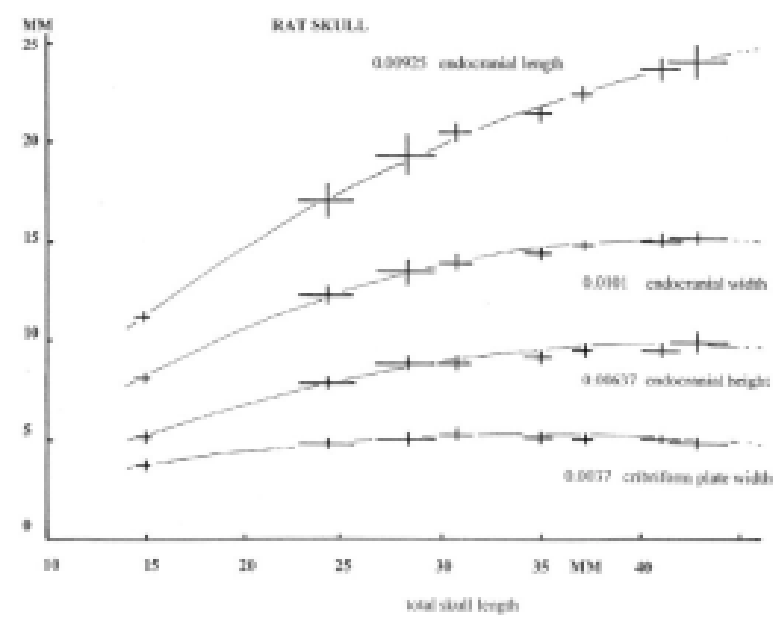

Fig. 1. Rat skull: four different measurements compared to the skull's total length. For all measurements the standard deviations are indicated; also indicated are the corresponding parabolic curves and quadratic factors.

figure; also added are the calculated quadratic factors: multiplied by two they give the growth reduction in $\mathrm{mm}$ of that distance for each $\mathrm{mm}$ of growth in length of the skull. For two measurements (endocranial width and height) the calculated curves suggest that the maximum is reached.

In Fig. 2 the greatest width in the rat skull (the bizygomatic width) is compared to the length of the same skull; also here the calculated curve is added showing that the growth in length of the skull is negatively influenced by the width.

\section{Cornish fowl}

In Fig. 3 the growth of the tarsometatarsus is compared to the growth of various body measurements; for three of them the results show a similar length at one week of age but a different increase during the following weeks; all measurements follow very closely the quadratic parabola added to the figure. Body depth that shows the greatest growth increase shows also the greatest reduction (per $\mathrm{cm}$ tarsometatarsus increase, body depth shows two times $0.07733 \mathrm{~cm}$ decrease: see Table 1). In Fig. 4 on the other hand, the dependence of the tarsometatarsus length on body weight is shown; also here the observed values match the calculated curve; the extrapolation of the curve suggests that, during the observations, the tarsometatarsus reached almost its maximum value and that from a weight of about $1.3 \mathrm{~kg}$ it will become shorter; this relationship seems logical as the tarsometatarsus bone has to support the weight of the chicken.

\section{Discussion}

From my study on nematodes (Geraert 1978a, 1978b, 1979) and from this study it is obvious that growth of one organ or body part has a definite relation to the growth of another organ or measurement. Whereas in the rat skull the different measurements are taken on exactly the same organ (so a close relationship is readily acceptable) the very close relationships for the
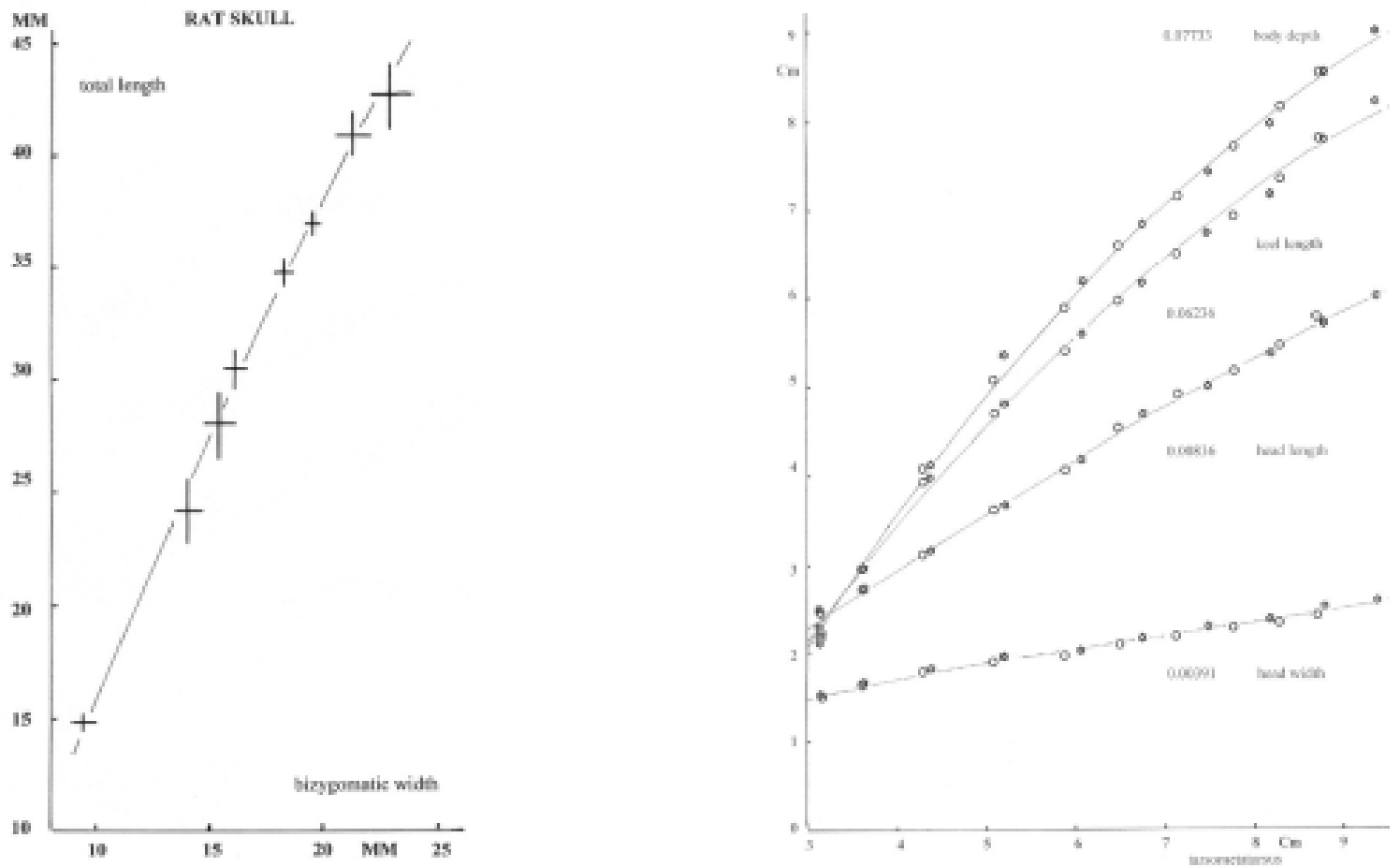

Fig. 2 (Left). Rat skull: total length compared to bizygomatic width. The eight measurements are represented by the standard deviations on either side of the means. The calculated parabolic curve is added.

Fig. 3 (Right). Dark cornish fowl: four different measurements compared to the tarsometatarsus length. Females are represented by circles, males by stars. Added are the parabolic curves and the quadratic factors. 


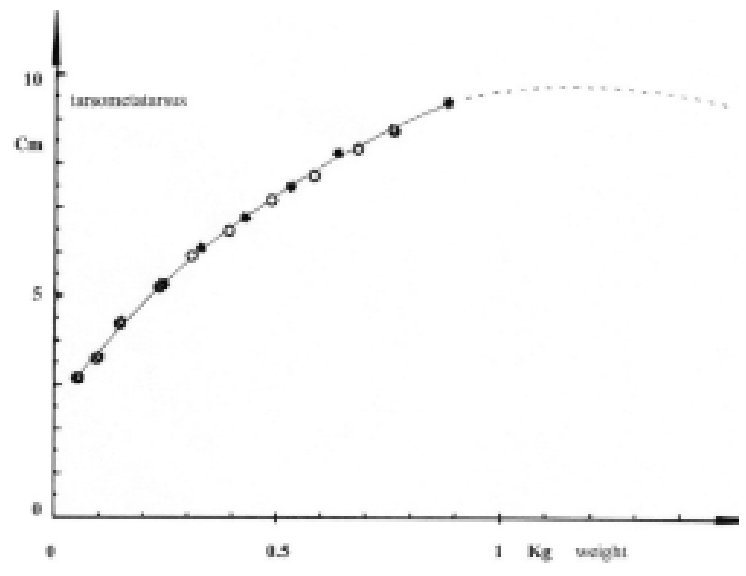

Fig. 4. Dark cornish fowl: tarsometatarsus compared to total weight. Females are represented by circles, males by stars; the extrapolation of the curve shows that the tarsometatarsus is theoretically almost at its maximum length.

various chicken parts more strongly support the theory presented here. All the curved lines, here shown, go to a theoretical maximum after which the structure or organ involved will (theoretically) no longer grow but will start to diminish; in one example the maximum is reached during the observations (rat skull: cribriform plate) and consequently the width of that plate diminishes. During growth in rat and chicken there is a mutual influence of the several body parts; in the rat skull, length influences negatively the growth of various skull measurements but the skull width influences negatively the growth in skull length; in the chicken the tarsometatarsus growth is reduced by weight (Fig. 4) but, on the other hand, several other measurements show growth reduction in relation to tarsometatarsus growth (Fig. 3). Figures 1 to 4 show only a selection; any measurement compared to any other measurement shows that one of them influences negatively the other. Theoretically such a growth reduction of one item compared to another item can be haphazard or can be regular; all the observations show that it is not only regular but also constant (e.g. Table 1 ). The only curve that is characterized by constant 'second differences' is a quadratic parabola; for all the measurements given here the appropriate curve has been calculated and added to the graph.

A quadratic curve has the general formula

$$
Y=a X^{2}+b X+c
$$

The values ' $b$ ' and ' $c$ ' in this formula have a mathematical meaning, not a biological one; they are needed to position the curve in a diagram; the most important factor is ' $a$ ': its negative sign shows that the $Y$-value decreases and its size shows exactly the degree of change in the growth of item $Y$ relative to $X$ (multiplied by two it gives the exact value of the second growth difference in $Y$ for one unit of $X$ ). By using this formula we assume that measurement $Y$ is the dependent variable and $X$ the independent variable but the examples given show that growth in general is more complicated, moreover the given measurements are probably not taken along the important growth axes. Nevertheless the negative sign of ' $a$ ' in various comparisons indicates why growth stops: it stops because the growth of a body item is constantly and negatively influenced by the growth of another body item. This does not happen suddenly or after some growth has occurred but it starts from the very beginning so I hypothesize that when growth starts a very precise growth pattern ensues that cannot be changed.

Growth is induced by growth promoting substances, and influenced by growth inhibiting substances. The factor 'a' could indicate the constantly changing relation between the production of growth promoting and growth inhibiting substances. The negative sign of this factor could indicate that the growth inhibiting substances become more and more important and are finally operative at the same level as the growth promoting substances: as a consequence growth ceases. It is also hypothesized that it is not because growth has ceased that the production of both substances ceases; the extrapolation of the obtained curves suggests that growth inhibiting substances become now constantly more important. If that is so, then the curve characterizes what is happening after growth: deterioration, malfunction and finally death. According to this speculation, the same metabolic processes that start when growth starts finally cause death.

What is presented here is a hypothesis that explains some morphometric observations; I do not know of any experimental

TABLE I

COMPARISON OF MEASURED AND CALCULATED VALUES FOR BODY DEPTH IN RELATION TO TARSOMETATARSUS IN CORNISH FOWL

\begin{tabular}{|c|c|c|c|c|c|}
\hline \multirow{3}{*}{$\frac{\text { Tarsometatarsus }=\mathbf{X}}{\text { Measured }}$} & \multicolumn{5}{|c|}{ Body depth = Y } \\
\hline & \multirow[t]{2}{*}{ Measured } & \multicolumn{4}{|c|}{ Calculated } \\
\hline & & & & Differences & Second diff. \\
\hline 3.16 & 2.29 & 2.30 & & & \\
\hline 3.17 & 2.32 & 2.32 & & & \\
\hline 3.62 & 2.99 & 2.99 & & & \\
\hline 3.64 & 2.98 & 3.02 & & & \\
\hline (4) & & & 3.536 & & \\
\hline 4.31 & 3.93 & 3.97 & & 1.330 & \\
\hline 4.4 & 3.96 & 4.09 & & & \\
\hline (5) & & & 4.866 & & -0.155 \\
\hline 5.1 & 5.12 & 4.99 & & & \\
\hline 5.24 & 5.36 & 5.16 & & 1.175 & \\
\hline 5.89 & 5.91 & 5.92 & & & \\
\hline (6) & & & 6.041 & & -0.154 \\
\hline 6.1 & 6.2 & 6.15 & & & \\
\hline 6.49 & 6.61 & 6.56 & & 1.021 & \\
\hline 6.77 & 6.85 & 6.84 & & & \\
\hline (7) & & & 7.062 & & -0.155 \\
\hline 7.15 & 7.18 & 7.20 & & & \\
\hline 7.51 & 7.41 & 7.53 & & 0.866 & \\
\hline 7.78 & 7.67 & 7.75 & & & \\
\hline (8) & & & 7.928 & & \\
\hline 8.22 & 7.94 & 8.10 & & & -0.154 \\
\hline 8.28 & 8.14 & 8.15 & & & \\
\hline 8.74 & 8.52 & 8.47 & & 0.712 & \\
\hline 8.8 & 8.52 & 8.51 & & & \\
\hline (9) & & & 8.640 & & \\
\hline 9.39 & 9 & 8.88 & & & \\
\hline
\end{tabular}

Measurements of females and males are mixed. In the first column the observed values are given together with the units in brackets. In the second column the corresponding observed values are given. In the third column the calculated $Y$-values are given for the corresponding $X$-value. In the fourth column the calculated $Y$-values for the units of $X$ are given. Column five shows the differences between the calculated values per $\mathrm{cm}$ tarsometatarsus increase. The last column shows the constant decrease of the increase. 
evidence that backs up this suggestion, neither can I suggest how experimentalists should test these ideas.

Slack's questions, mentioned in the introduction, could have the following answers: (1) The absolute size of the whole is controlled by the constantly changing equilibrium between the various growth promoting and growth inhibiting substances; during evolution the changing in that equilibrium can augment producing bigger men or mice; (2) The constancy of proportions of individual parts is maintained by an active process that controls the exact amount of the substances that control growth; (3) Allometry is produced by the constant second differences in one growth parameter in relation to another growth parameter; it is not so that various parts of an organism grow at a constant relative rate (as mentioned by Bartoletti et al., 1999) but at a constantly changing relative rate.

\section{Material and Methods}

Two studies were finally used: (1) Ford and Horn (1959) on the skull of 40 albino rats, of which groups of five were killed at birth, at ten, fifteen, twenty, thirty, forty, sixty and eighty days of age; immediately after death radiographs were taken of each rat on which various measurements to 0.1 $\mathrm{mm}$ were made; (2) Kidwell and Williams (1956) on a chicken variety (Dark Cornish fowl) present in Louisiana State University; 83 males and 101 females were followed during ten weeks; six measurements were taken on each at one week of age and at weekly intervals thereafter; weights were taken to the nearest gram, measurements to the nearest $\mathrm{mm}$.

\section{Conclusions}

1. Growth is better considered as a uniformly decelerated movement rather than as an exponential process.

2. Allometry can best be described by a polynomial equation of the second degree resulting in a parabola (= quadratic curve); the quadratic factor gives the exact change in growth of the dependent variable for each unit of growth of the independent variable. At the apex of the parabola the growth of the dependent variable stops and is followed by negative growth.
3. Growth, adulthood, ageing and death could be aspects of exactly the same process that starts from the very beginning of multicellularity; it seems that growth is influenced by specific growth promoting and growth inhibiting substances; it is hypothesized that growth inhibiting substances become more and more important so that growth stops, deterioration continues and death follows.

\section{References}

ALBERTS, B., JOHNSON, A., LEWIS, J., RAFF, M., ROBERTS, K. and WALTER, P. (2002). Molecular Biology of The Cell. Garland Science, 1463 pp.

BARTOLETTI, S., FLURY, B.D. and NEL, D.G. (1999). Allometric extension. Biometrics 55: 1210-1214.

FORD, E.H.R. and HORN, G. (1959). Some problems in the evaluation of differential growth in the rat skull. Growth 23: 191-204.

GERAERT, E. (1978a). On growth and form in Nematoda: oesophagus and body width in Tylenchida. Nematologica 24: 137-158.

GERAERT, E. (1978b). On growth and form in Nematodes: II. Oesophagus and body width in Dorylaimida. Nematologica 24: 347-360.

GERAERT, E. (1979). Growth and form in Nematodes: III. Comparison of oesophagus and body shape. Nematologica 25: 1-21.

HUXLEY, J.S. (1924). Constant differential growth-ratios and their significance. Nature 114: 895-896.

KIDWELL, J.F.and WILLIAMS, E. (1956). Allometric growth of the dark Cornish Fowl. Growth 20: 275-293.

SLACK, J.M.W. (1999). Problems of Development: The Microcosm and the Macrocosm. In On Growth and Form: Spatio-temporal Pattern Formation in Biology. Eds. Chaplain, M.A.J., Singh, G.D. and McLachlan, J.C. New York. John Wiley \& Son. p. 1-12.

WEST, G.B., BROWN, J.H. and ENQUIST, B.J. (1997). A General Model for the Origin of Allometric Scaling Laws in Biology. Science 276: 122-133.

WEST, G.B., BROWN, J.H. and ENQUIST, B.J. (2001). A general model for ontogenetic growth. Nature 413: 628-631.

ZEGER, S.L. and HARLOW, S.D. (1987). Mathematical models from laws of growth to tools for biological analysis: fifty years of Growth. Growth $51: 1-21$.

Received: November 2003

Reviewed by Referees: December 2003

Modified by Authors and Accepted for Publication: February 2004 